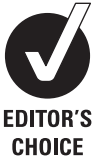

CHOICE

\title{
Care from health visitors trained in psychological intervention methods may prevent depression in mothers not depressed 6 weeks postnatally
}

\section{Patricia Leahy-Warren, ${ }^{1}$ Paul Corcoran ${ }^{2}$}

10.1136/ebn.2011.100085

${ }^{1}$ Catherine McAuley School of Nursing and Midwifery, University College Cork,

Cork, Ireland

${ }^{2}$ The National Suicide Research Foundation, Cork, Ireland

\section{Correspondence to:} Patricia Leahy-Warren Catherine McAuley School of Nursing and Midwifery, Brookfield Health Sciences Complex, University College Cork, Ireland;

patricia.leahy@ucc.ie

\section{Commentary on: Brugha TS, Morrell CJ, Slade P, et al. Universal prevention of depression in women postnatally: cluster randomized trial evidence in primary care. Psychol Med 2011;41:739-48.}

\section{Implications for practice and research}

- Nurses and midwives who provide postnatal care need to receive training in identification and psychological intervention methods to prevent depression.

- They will need to provide more visits to all mothers within the first 12-month postdelivery.

- Further randomised controlled trials (RCTs) in this area should utilise midwives/health visitors (HVs) as the administrators of the intervention.

- They should also seek control for the increased number of contacts associated with interventions of this nature.

- There is a need for further RCTs in postnatal care to disentangle the effects of perceived support, the therapeutic relationship and the psychological intervention.

\section{Context}

Approximately $13 \%$ of mothers experience postnatal depression (PND). ${ }^{1}$ It occurs at a crucial time in a mother's life, can persist for long periods and can have adverse effects on her partner and on the emotional and cognitive development of infants and children. Reported prevalence rates of PND vary within and across countries and range from $4.4 \%$ at 12 -month postdelivery to $73.7 \%$. This substantial variation may be attributed to measurements used, sampling methodologies, sociodemographic factors, parity, timescale of the study and cultural diversity. ${ }^{2}$

\section{Methods}

This study tested whether receiving care from a HV trained in identification and psychological intervention methods prevents depression 6-18 months postnatally in women who are not depressed 6 weeks postnatally. The study was a large prospective cluster trial randomised by GP practice in 101 primary care teams in the UK. Eligible women in the intervention group (IG) practices treated by $\mathrm{HV}$ s trained in cognitive behavioural therapy (CBT) and person centred care (PCC) were compared with women in care as usual (CAU) practices. The HVs were blind to the main outcome measure of PND (Edinburgh Postnatal Depression Scale (EPDS) $\geq 12$ ). Outcome comparisons at 6-, 12- and 18-month follow-up were carried out on an intention-to-treat basis and adjusted for individual level variables and baseline measures.

\section{Findings}

Risk of PND at 6 months was significantly lower (adjusted OR $0.71,95 \%$ CI 0.53 to $0.97, p=0.031$ ) in the IG. A similar effect was observed for both types of intervention (CBT and PCC) and in women with low- and subthreshold baseline depression scores.

\section{Commentary}

The study findings are likely to be generalisable beyond the UK. They build on previous findings, demonstrating that psychosocial interventions by healthcare professionals may be more effective postnatally when integrated into routine visits. ${ }^{34}$ We recommend that, in future research in this area, healthcare professionals administer the psychosocial interventions. An innovative aspect of this study is the focus on women who screen negative for depression postnatally and the demonstration that the intervention was as effective for subthreshold women as it was for those with very low depression scores on the EPDS. The findings build on a previous publication ${ }^{5}$ from the same study, which demonstrated a preventive effect of the intervention on women who screened positive for depression postnatally.

In the methods section, the description of the randomisation is unclear. There is a suggestion that the primary care practices were randomised and then HVs within these practices were also randomised. It is difficult to disentangle the methods specifically related to this article from those related to the previously published article. ${ }^{5}$ The effect of the intervention was adjusted for living alone and 'any life events'. There is a lack of detail regarding these life events, and there was no assessment of social support that has been previously found to be having a role in prevention of PND. ${ }^{6}$ There was no difference between CBT and PCC in effectiveness. Both involved more contacts than did CAU. Therefore, research needs to address the question of whether it is the nature of the intervention or number of contacts made that causes the positive effect. The authors' use of the term Universal in their conclusion seems inappropriate given that the focus was on women who screened negative for depression. Furthermore, the term Universal prevention of depression in the title could be misinterpreted as to mean eradication of PND. Nevertheless, this is an important study with implications for policy and practice in relation to PND.

Competing interests None. 


\section{References}

1. 0' Hara M, Swain A. Rates and risk of postpartum depression-a meta-analysis. Int Rev Psychiatr 1996;8:37-54.

2. Leahy-Warren P, McCarthy G. Postnatal depression: prevalence, mothers' perspectives, and treatments. Arch Psychiatr Nurs 2007;21:91-100.

3. MacArthur C, Winter HR, Bick DE, et al. Effects of redesigned community postnatal care on womens' health 4 months after birth: a cluster randomised controlled trial. Lancet 2002;359:378-85.

4. Dennis CL. Psychosocial and psychological interventions for prevention of postnatal depression: systematic review. BMJ 2005;331:15.
5. Morrell CJ, Slade P, Warner R, et al. Clinical effectiveness of health visitor training in psychologically informed approaches for depression in postnatal women: pragmatic cluster randomised trial in primary care. $B M J$ 2009;338:a3045.

6. Leahy-Warren P, McCarthy G, Corcoran P. Postnatal depression in first-time mothers: prevalence and relationships between functional and structural social support at 6 and 12 weeks postpartum. Arch Psychiatr Nurs 2011;25:174-84. 